This item was submitted to Loughborough's Research Repository by the author.

Items in Figshare are protected by copyright, with all rights reserved, unless otherwise indicated.

\title{
Model based robust control approach for batch crystallization product design
}

PLEASE CITE THE PUBLISHED VERSION

PUBLISHER

(C) Elsevier

VERSION

AM (Accepted Manuscript)

LICENCE

CC BY-NC-ND 4.0

\section{REPOSITORY RECORD}

Nagy, Zoltan K.. 2009. "Model Based Robust Control Approach for Batch Crystallization Product Design". figshare. https://hdl.handle.net/2134/5658. 
This item was submitted to Loughborough's Institutional Repository (https://dspace.lboro.ac.uk/) by the author and is made available under the following Creative Commons Licence conditions.

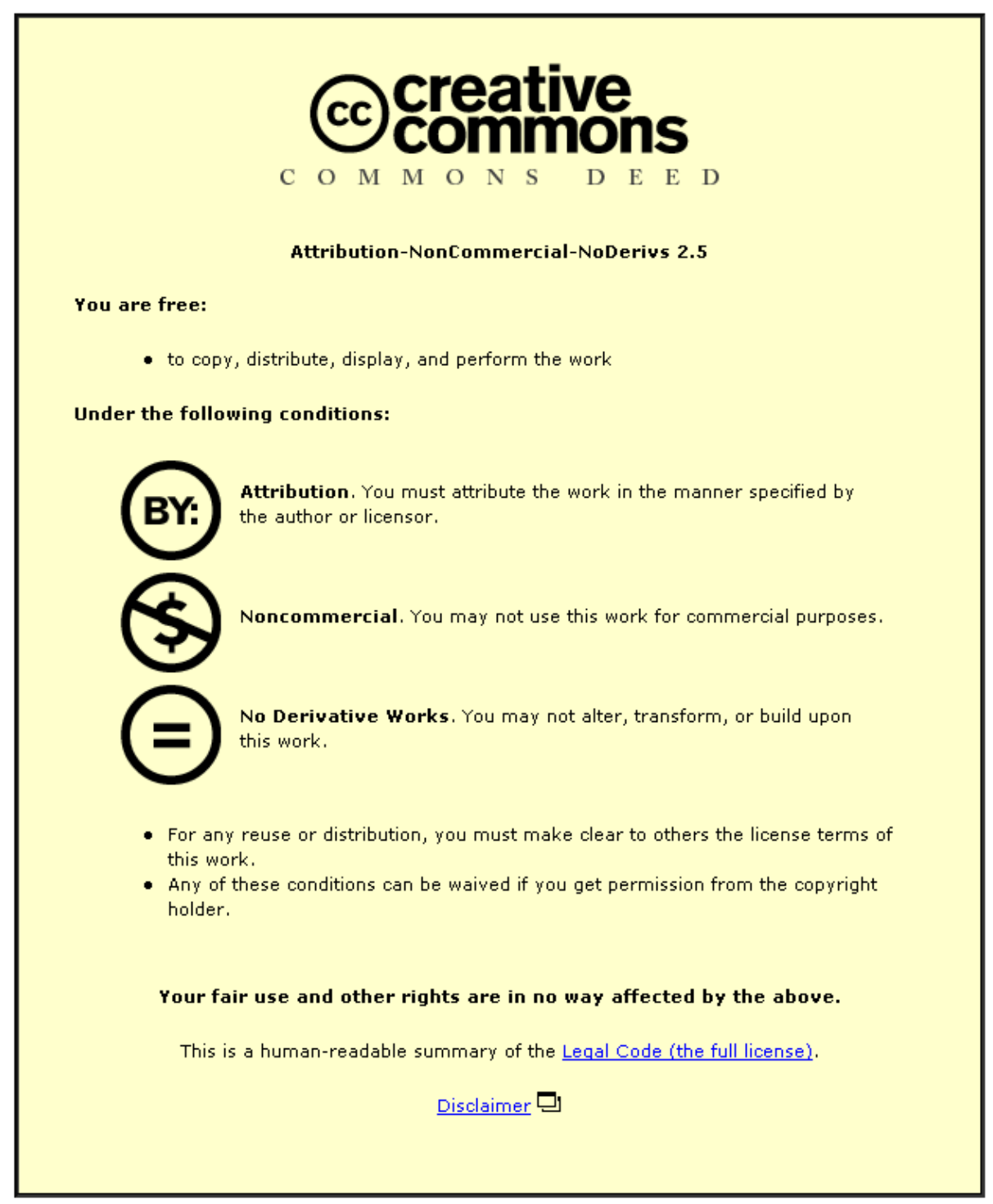

For the full text of this licence, please go to: http://creativecommons.org/licenses/by-nc-nd/2.5/ 


\title{
Model Based Robust Control Approach for Batch
}

\section{Crystallization Product Design}

\author{
Zoltan K. Nagy ${ }^{1}$ \\ Chemical Engineering Department, Loughborough University, \\ Loughborough, LE11 3TU, United Kingdom
}

\begin{abstract}
The paper presents a novel control approach for crystallization processes, which can be used for designing the shape of the crystal size distribution to robustly achieve desired product properties. The approach is based on a robust optimal control scheme, which takes parametric uncertainties into account to provide decreased batch-to-batch variability of the shape of the crystal size distribution. Both open-loop and closed loop robust control schemes are evaluated. The open-loop approach is based on a robust end-point nonlinear model predictive control (NMPC) scheme which is implemented in a hierarchical structure. On the lower level a supersaturation control approach is used that drives the system in the phase diagram according to a concentration versus temperature trajectory. On the higher level a robust model-based optimization algorithm adapts the setpoint of the supersaturation controller to counteract the effects of changing operating conditions. The process is modelled using the population balance equation (PBE), which is solved using a novel efficient approach that combines the quadrature method of moment (QMOM) and method of characteristics (MOC). The proposed robust model based control approach is corroborated for the case of various desired shapes of the target distribution.
\end{abstract}

Keywords: Crystallization control, batch nonlinear model predictive control, robust optimal control, population balance model, distribution shaping control, quadrature method of moments, method of characteristics.

\footnotetext{
${ }^{1}$ E-mail: Z.K.Nagy@lboro.ac.uk
} 


\section{Introduction}

Crystallization is one of the key unit operations in the pharmaceutical, food and fine chemicals industries. Despite the long history and widespread application of batch crystallization, there remains a disproportionate number of problems associated with its control (Braatz, 2002), mainly related the complex nonlinear dynamics with nonideal mixing, and various disturbances characteristic to these systems. The operating conditions of the crystallization process determine the physical properties of the products which are directly related to the crystal size distribution (CSD), shape or polymorphic form (Larsen et al., 2006; Braatz and Hasebe, 2002). These properties determine the efficiency of downstream operations, such as filtration, drying, and tablet formation, and the product effectiveness, such as bioavailability and shelf-life. With the recent change of industrial procedures from Quality-byTesting $(\mathrm{QbT})$ to Quality-by-Design $(\mathrm{QbD})$ and the advent of process analytical technology (PAT) initiative, especially in the pharmaceutical industries (Barett et al., 2005; Yu et al., 2004), approaches which can be used to design desired product properties are of great interest (Fujiwara et al., 2005; Hounslow and Reynolds, 2006; Wibovo and Chang, 2001). The classical control objectives expressed in characteristics of the size distribution (e.g. maximize average size, minimize coefficient of variation) can lead to conservative and economically inefficient designs of the crystallization systems. The paper presents an approach which can be used to directly design the shape of the crystal size distribution to achieve desired product properties. The method is able for example to minimize filtration time without generating unnecessarily large crystals. Since dissolution rate depends on the shape of the CSD, when the resulting crystals represent the final product (e.g. drugs for inhalers) controlling the shape of the CSD can provide novel applications in the area of drug delivery, or environmentally friendly dosage of pesticides, where particular multimodal distributions can be designed to achieve desired concentration level of the active ingredient. The crystallization system is modelled via the population balance equation (PBE) which is directly used in the optimization procedure where the objective function is expressed in terms of the shape of the entire CSD. The population balance equation (PBE) is solved using a novel approach based on the combination of the quadrature method of moments (QMOM) (McGraw, 1997) and method of characteristics (LeVeque, 1992). Crystallization models are generally subject to significant uncertainties. Several novel 
approaches have been proposed recently in the literature for incorporating robustness in the control of crystallisation processes (Nagy and Braatz, 2004; Nagy and Braatz, 2003), including the application to the control of polymorphic transformations (Hermanto et al., 2007). A robust optimization based approach is evaluated and it is shown that taking parametric uncertainties into account in the problem formulation can lead to significant improvement in the robustness of the prodcut quality. The control approach is implemented in a hierarchical structure where on the lower level a model-free crystallization control methodology, the supersaturation controller, drives the system in the phase diagram, rather then in the time domain, whereas on the higher level a robust on-line model based optimization algorithm, the so-called distributional batch nonlinear model predictive control (NMPC), adapts the setpoint of the supersaturation controller to counteract the effects of changing operating conditions (Rawlings et al., 1993; Nagy and Braatz, 2003; Nagy et al., 2008). The optimization problem is solved using an efficient multistage approach implemented in the optimization package OptCon (Nagy et al., 2004; Nagy et al., 2007). The proposed approach is corroborated in the case of a simulated crystallization system. The simulation results are supported by results from initial laboratory experiments. The practical experiments illustrate the importance of judicious combination of state-ofthe-art PAT tools and efficient optimization algorithms for the successful implementation of the online model based control approach.

\section{Population balance modelling of batch crystallization processes}

Considering a single growth direction with one characteristic length $L$, and a well-mixed crystallizer with growth and nucleation as the only dominating phenomena the crystal size distribution (CSD) expressed in the number density function $f_{n}(L, t)$, is given by the population balance equation (PBE) with the form

$$
\frac{\partial f_{n}(L, t)}{\partial t}+\frac{\partial\left\{G\left(S, L ; \theta_{g}\right) f_{n}(L, t)\right\}}{\partial L}=B\left(S ; \theta_{b}\right) \delta\left(r_{0}, L\right),
$$

with initial condition given by the size distribution of seed, $f(L, 0)=f_{\text {seed }}\left(L_{0}\right), t$ is time, $G\left(S, L ; \theta_{g}\right)$ is the rate of crystal growth. $B\left(S ; \theta_{b}\right)$ is the nucleation rate, $S=C-C_{\text {sat }}$ is the supersaturation, $C$ is the 
solute concentration, $C_{s a t}(T)$ is the saturation concentration at the temperature $T$, and $\theta_{g}$ and $\theta_{b}$ are vectors of growth and nucleation kinetic parameters, respectively. The partial differential equation can be reduced to a system of ODEs by applying a combination of the method of characteristics (MOC) and quadrature method of moments (QMOM). The aim of the MOC is to solve the PBE by finding characteristic curves in the $L-t$ plane that reduce the PBE to a system of ODEs. The $L-t$ plane is expressed in a parametric form by $L=L(\mathcal{Z})$ and $t=t(\mathcal{Z})$, where the parameter $\mathcal{Z}$ gives the measure of the distance along the characteristic curve. Therefore, $f_{n}(L, t)=f_{n}(L(\mathcal{Z}), t(\mathcal{Z}))$, and applying the chain rule gives,

$$
\frac{d f_{n}}{d \mathcal{Z}}=\frac{d L}{d \mathcal{Z}} \frac{\partial f_{n}}{\partial L}+\frac{d t}{d \mathcal{Z}} \frac{\partial f_{n}}{\partial t}
$$

Comparing (2) with (1) we find $\mathcal{Z}=t$ and the characteristic equations can be derived. Solving these together with the system of equations which results by applying the QMOM, we can calculate the dynamic evolution of $f_{n}(L, t)$ by the following ODEs,

$$
\begin{aligned}
\frac{d \mu_{0}}{d t} & =B \\
\frac{d \mu_{j}}{d t} & =j \sum_{i=1}^{N_{q}} w_{i} L_{i}^{j-1} G\left(L_{i}\right)+B r_{0}^{j}, \quad j=1,2,3 \\
\frac{d L}{d t} & =G \\
\frac{d f_{n}}{d t} & =-f_{n}(L, t) \frac{d G}{d L}+B \delta\left(r_{0}, L\right)
\end{aligned}
$$

with initial conditions $x_{0}=\left[\mu_{0}(0), \ldots, \mu_{3}(0), L_{0}, f_{\text {seed }}\left(L_{0}\right)\right]$ where the $j$ th moment $\mu_{j}$ and its quadrature approximation is defined by

$$
\mu_{k}=\int_{0}^{+\infty} f_{n}(L) L^{k} d L \approx \sum_{i=1}^{N_{q}} w_{i} L_{i}^{k}, \quad k=0,1, \ldots, \infty
$$

The number of quadrature points $\left(N_{q}\right)$ and the corresponding weights $\left(w_{i}\right)$ and abscissas $\left(L_{i}\right)$ can be determined through the product-difference (PD) algorithm (McGraw, 1997) or via direct solution of a differential-algebraic (DAE) system that results by setting the condition of no error if the integral from the moment definition is replaced with its quadrature approximation. More details on the solution algorithm can be found in Aamir et al. (2008). The solute concentration is given by

$$
C(t)=C(0)-k_{v} \rho_{c}\left(\mu_{3}(t)-\mu_{3}(0)\right)
$$


where $\rho_{c}$ is the density of crystals and $k_{v}$ the volumetric shape factor. Several models for growth and nucleation kinetics have been developed and are available in the literature (Garside, 1984; Nyvlt et al., 1985; Rawlings et al., 1993 . The most common kinetic models is given by

$$
B=k_{b} S^{b},
$$

for primary nucleation and by

$$
B=k_{b} S^{b} \mu_{3},
$$

for secondary nucleation mechanisms when nuclei form from existing crystals, with nucleation parameters $\theta_{b}=\left[k_{b}, b,\right]$. The apparent effect of crystal size on the growth rate is often described by the empirical expressions (Jones, 2002) of the form,

$$
G=k_{g} S^{g}(1+\gamma L)^{p},
$$

with growth parameters $\theta_{g}=\left[k_{g}, g, \gamma, p\right]$. The expression given by eq.(8) is incorporated in the model used in this study to describe the widening effect of the CSD observed experimentally. The dissolution mechanism is also included in the model and is solved similarly, considering similar size dependence and dissolution rate about two times faster than the growth. The model equations are similar in both cases and the growth expression is switched to dissolution when the solution becomes under-saturated $(S<0)$.

Solving the system of ODEs (3) with different initial conditions obtained by varying $L_{0}$, the shape of the distribution can be obtained with desired resolution. Note that the proposed solution method can be used for the efficient solution of generic PBEs, including the case when agglomeration or breakage mechanisms are also considered together with nucleation and growth.

Several approaches have been proposed for designing the operating curves for crystallization systems. Generally speaking, two main categories can be distinguished, (i) the model-based approach (Rawlings et al., 1993; Sheikhzadeh et al., 2008a; 2008b; Shakar et al., 2006; Ward et al., 2006; Worlitschek and Mazzotti, 2004) and (ii) the direct design (Fujiwara et al., 2005; Zhou et al., 2006; Nagy et al., 2008). In the model-based design approach the detailed model (3) is used together with optimization techniques to determine temperature versus time trajectories (Braatz, 2002), which 
optimize desired product properties, usually expressed as functions of the moments of the CSD. The direct design approach is based on the understanding of the basic concept of crystallization, to operate the system within the metastable zone bounded by the nucleation and solubility curves (see Figure 1). In this technique a supersaturation setpoint profile is chosen experimentally based on the application of a wide range of PAT-based approaches (Fujiwara et al., 2002; Barrett and Glenon, 2002), and it is followed in the phase diagram using a supersaturation controller based on concentration measurement. The direct design approach can be implemented for both seeded and un-seeded operations. Seeded operation is often used in practice to try to avoid uncertainties related to the metastable zonce width (MSZW). However the generation of high quality seed with uniform and reproducible CSD is in general difficult and hence variability in seed CSD can lead to variations in the product CSD. When consistent in situ seed generation is a possibility via controlled primary nucleation un-seeded operation is preferred. The approach proposed in the paper combines the concept of the model-free direct design approach with the model-based method in a hierarchical control algorithm, in which a model-based robust optimization determines the operating profile in the phase diagram, which is used then as the setpoint for the supersaturation controller.

\section{Distributional robust batch NMPC for end-point property control}

The optimal control problem to be solved off-line or on-line in every sampling period in the control algorithm (Nagy and Braatz, 2003) can be formulated as follows:

$$
\min _{u \in \mathcal{U}} \mathcal{H}(x, u ; \theta)
$$

subject to:

$$
\begin{aligned}
& \dot{x}(t)=f(x(t), u(t) ; \theta), \\
& y(t)=g(x(t), u(t) ; \theta), \\
& x\left(t_{k}\right)=\hat{x}\left(t_{k}\right), \quad x\left(t_{0}\right)=\hat{x}_{0}, \\
& h(x(t), u(t) ; \theta) \leq 0, \quad t \in\left[t_{k}, t_{F}\right],
\end{aligned}
$$

where $\mathcal{H}$ is the performance objective, $t$ is the time, $t_{k}$ is the time at sampling instance $k, t_{F}$ is the final 
time at the end of prediction, $x(t) \in \mathbb{R}^{n_{x}}$ is the vector of states, $u(t) \in \mathcal{U} \subset \mathbb{R}^{n_{u}}$ is the set of input vectors, $y(t) \in \mathbb{R}^{n_{y}}$ is the $n_{y}$ vector of measured variables used to compute the estimated states $\hat{x}\left(t_{k}\right)$, $\theta \in \Theta \subset \mathbb{R}^{n_{\theta}}$ is the $n_{\theta}$ vector of uncertain parameters, where the set $\Theta$ can be either defined by hard bounds or probabilistic, characterized by a multivariate probability density function. The function $f: \mathbb{R}^{n_{x}} \times \mathcal{U} \times \Theta \rightarrow \mathbb{R}^{n_{x}}$ is the twice continuously differentiable vector function of the dynamic equations of the system, $g: \mathbb{R}^{n_{x}} \times \mathcal{U} \times \Theta \rightarrow \mathbb{R}^{n_{y}}$ is the measurement equations function, and $h: \mathbb{R}^{n_{x}} \times \mathcal{U} \times \Theta \rightarrow \mathbb{R}^{c}$ is the vector of functions that describe all linear and nonlinear, time-varying or end-time algebraic constraints for the system, where $c$ denotes the number of these constraints. The objective function can have the following general form:

$$
\mathcal{H}(x(t), u(t) ; \theta)=\mathcal{M}\left(x\left(t_{F}\right) ; \theta\right)+\int_{t_{k}}^{t_{F}} \mathcal{L}(x(t), u(t) ; \theta) d t .
$$

We assume that $\mathcal{H}: \mathbb{R}^{n_{x}} \times \mathcal{U} \times \Theta \rightarrow \mathbb{R}$ is twice continuously differentiable. The objective function $\mathcal{H}$ consists of a terminal cost function, $\mathcal{M}: \mathbb{R}^{n_{x}} \times \Theta \rightarrow \mathbb{R}$, and a running cost function, $\mathcal{L}: \mathbb{R}^{n_{x}} \times \mathcal{U} \times \Theta \rightarrow \mathbb{R}$. The form of (14) is general enough to express a wide range of objectives encountered in NMPC applications (moving or shrinking horizon approach on regulation and/or setpoint tracking problems, direct minimization of the operation time, optimal initial conditions, multiple simultaneous objectives, treatment of soft constraints, terminal penalty term for stability conditions, etc.).

The repeated optimization problem is solved by formulating a discrete form, that can be handled by conventional solvers (Biegler and Rawlings, 1991). The batch time $t \in\left[0, t_{f}\right]$ is divided into $N$ equally spaced time intervals $\Delta t$ (stages), with discrete time steps $t_{k}=k \Delta t$, and $k=0,1, \ldots, N$. The NMPC approach is implemented in the Matlab toolbox, OptCon (Nagy at al., 2004), which is based on a state-of-the-art large-scale nonlinear optimization solver (HQP) (Franke et al.), which uses a multiple shooting algorithm (Diehl et al., 2002). The main idea of the shrinking horizon on-line control algorithm is illustrated in Figure 2.

Consider the case of parameter uncertainty, with $\delta \theta \in \mathbb{R}^{n_{\theta}}$ defined as the perturbation about the 
nominal parameter vector $\hat{\theta}$. The real uncertain parameter vector is then given by $\theta=\hat{\theta}+\delta \theta$. Assuming zero mean, normal measurement errors, and known covariance matrix, the set of possible parameter values is given by the hyperellipsoidal confidence region, defined as

$$
\Theta(\alpha) \triangleq\left\{\theta:(\theta-\hat{\theta})^{T} \mathbf{V}_{\theta}^{-1}(\theta-\hat{\theta}) \leq \chi_{n_{\theta}}^{2}(\alpha)\right\},
$$

where $\alpha$ is the confidence level, $\chi_{n_{\theta}}^{2}(\alpha)$ is a quantile of the chi-squared distribution with $n_{\theta}$ degrees of freedom, and $\mathbf{V}_{\theta} \in \mathbb{R}^{n_{\theta} \times n_{\theta}}$ is the parameter covariance matrix. Uncertainty description (15) results most commonly from typical least-squares identification procedures from experimental data. We denote with $\psi\left(x\left(t_{f}\right) ; \theta\right)$ the end-point property of interest. Considering the mean-variance approach the following objective function is used to account for parameter uncertainties in the NMPC:

$$
\mathcal{H}=(1-w) \mathcal{E}\left[\psi\left(x\left(t_{f}\right), \theta\right)\right]+w V_{\psi}\left(t_{f}\right)
$$

where $\mathcal{E}$ and $V_{\psi} \in \mathbb{R}$ is the expected value and variance, respectively, of the property at the end of the batch, and $w \in[0,1]$ is a weighting coefficient that quantifies the tradeoff between nominal and robust performance. The main advantage of this approach compared to the classical minmax optimizations is that the tradeoff between nominal and robust performance can be controlled by appropriately weighting the two objectives. Expected value and variance can be computed efficiently using a second order power series expansion,

$$
\delta \psi=L \delta \theta+\frac{1}{2} \delta \theta^{T} \mathbf{M} \delta \theta+\ldots,
$$

where $L=(d \psi / d \theta)_{\hat{\theta}, u} \in \mathbb{R}^{n_{\theta}}$, and $\mathbf{M}=\left(d^{2} \psi / d \theta^{2}\right)_{\hat{\theta}, u} \in \mathbb{R}_{n_{\theta} \times n_{\theta}}$ are the first and second order sensitivities, respectively. Assuming zero mean, normally distributed parameters $\delta \theta$, deriving the expected value and variance of $\delta \psi$ based on (17) gives the analytical expressions:

$$
\begin{aligned}
& \mathcal{E}[\delta \psi]=\frac{1}{2} \operatorname{tr}\left(\mathbf{M V}_{\theta}\right) \\
& V_{\psi}=L \mathbf{V}_{\theta} L^{T}+\frac{1}{2}\left[\operatorname{tr}\left(\mathbf{M V}_{\theta}\right)\right]^{2}
\end{aligned}
$$

where $\operatorname{tr}(\mathbf{A})$ is the trace of matrix $\mathbf{A}$. The feasibility of the optimization under parametric uncertainty is achieved by reformulating the constraints in a probabilistic sense: 


$$
\mathbb{P}\left(h_{i}(x, u ; \theta) \leq 0\right) \geq \alpha_{i}
$$

where $\mathbb{P}$ is the probability and $\alpha_{i}$ is the desired confidence level for the satisfaction of constraint $i$. The robust formulation of (20) can be written using the t-test in the form:

$$
\mathcal{E}\left[h_{i}\right]+t_{\alpha / 2, n_{\theta}} \sqrt{V_{h_{i}}} \leq 0, \quad i=1, \ldots, c .
$$

The expected value $\left(\mathcal{E}\left[h_{i}\right]\right)$ and covariance $\left(V_{h_{i}}\right)$ of the constraint $h_{i}$ can be evaluated using first or second order approximations. For first order approximation $\mathcal{E}\left[h_{i}(x, u ; \theta)\right]=h_{i}(x, u ; \hat{\theta})$ and $V_{h_{i}}=L_{h_{i}} \mathbf{V}_{\theta} L_{h_{i}}^{T}$, whereas for second order approximation expressions similar to (18) and (19) can be used, with $L_{h_{i}}=\left(d h_{i} / d \theta\right)_{\hat{\theta}, u} \in \mathbb{R}^{n_{\theta}}$, and $\mathbf{M}_{h_{i}}=\left(d^{2} h_{i} / d \theta^{2}\right)_{\hat{\theta}, u} \in \mathbb{R}^{n_{0} \times n_{\theta}}$. In this formulation the algorithm shows robust performance in the sense of constraint satisfaction and decreased variance of the performance index.

\section{Application of the robust optimal control and batch NMPC for crystallization product design}

For the case studies the crystallization of a proprietary pharmaceutical in water/isopropanol mixture was considered as the model system, for which nucleation and growth kinetics were determined experimentally. The solubility of the compound in water/isopropanol is given by,

$$
C_{\text {sat }}(T)=3.28 \cdot 10^{-5} T^{2}-1.10 \cdot 10^{-3} T+0.02
$$

where $C_{\text {sat }}$ is the solubility in $\mathrm{g} / \mathrm{g}$ solvent and $T$ is the temperature in ${ }^{\circ} \mathrm{C}$.

It was found that for the system exhibits a size dependent growth of the form (8), hence the shape of the CSD changes significantly during the batch. The growth kinetics vector is given by $\theta_{g}=\left[4.34 \mu m s^{-1}, 1.27,0.003 \mu m^{-1}, 1.23\right]$ and for nucleation $\theta_{b}=\left[6.56 \cdot 10^{-6} \mu m^{-3} s^{-1}, 3.8\right]$. Different product design problems were considered, when various objective functions expressed as desired shapes of the CSD were optimized and the required temperature profiles were determined. The novel feature of the proposed approach is that the optimization is performed in the phase diagram, and a concentration trajectory as a function of temperature $C_{\text {set }}=f(T)$ is obtained. This allows the direct 
application of the widely accepted supersaturation control in conjunction with the NMPC. The optimization problem can be expressed by the generic robust formulation:

$$
\begin{array}{r}
\min _{C_{\text {set }}(T)}\left\{(1-w) \sum_{k}\left(f_{n}\left(L_{k}, t_{f} ; \theta\right)-f_{n}^{\text {desired }}\left(L_{k}, t_{f}\right)\right)^{2}\right. \\
+w V\left[f_{n}\left(L, t_{f} ; \theta\right]\right\} \\
\text { s.t. } \quad T_{\min } \leq T(t) \leq T_{\max } \\
R_{\min } \leq \frac{d T}{d t} \leq R_{\max } \\
C\left(t_{f}\right) \leq C_{\max }
\end{array}
$$

where $f_{n}^{\text {desired }}\left(L_{k}, t_{f}\right)$ is the desired (setpoint) CSD with a given shape at the end of the batch, $T_{\min }=21{ }^{\circ} \mathrm{C}, T_{\max }=50{ }^{\circ} \mathrm{C}, R_{\min }=-1.2{ }^{\circ} \mathrm{C} / \mathrm{min}, R_{\max }=0.15{ }^{\circ} \mathrm{C} / \mathrm{min}$ are the operating constraints determined by the bounds and minimum and maximum rate changes of the temperature profiles, respectively, $C$ is the concentration and $C_{\max }=0.015 \mathrm{~g} / \mathrm{g}$ solvent is the maximum concentration at the end of batch required to achieve a desired crystallization yield. The values specified were used for most of the studies presented in the paper. For most crystallization processes there are significant variations in the metastable zone width (MSZW), which is incorporated in the optimization by considering uncertainties in the nucleation parameters $\left(\theta_{b}=\left[k_{b}, b\right]\right)$. Figure 3 shows the metastable zone width in the phase diagram delimited by the solubility and nucleation curves. Because of the uncertainties in the nucleation kinetics there is a nucleation region, with a width given by the $99 \%$ confidence intervals obtained via Monte Carlo simulations. The operating profiles resulted by solving (23)-(24) for the nominal case $(w=0)$ and one robust case $(w=0.3)$ are shown in the phase diagram in Figure 3. In the first case the target CSD was monomodal with a shape biased toward larger particles to improve filtration, obtained using a lognormal distribution $f_{n}^{\text {desired }}(L)=1 /(\sqrt{2 \pi} L \sigma) \exp \left(-0.5((\ln (L)-\bar{L}) / \sigma)^{2}\right)$ with mean of $\bar{L}=-6$ and standard deviation of $\sigma=0.22$ with units of the distribution in $\mathrm{m}^{-1}$. The robust operating profile corresponds to a trajectory, which is further away from the nucleation zone throughout the entire batch. The operating profiles are implemented using a supersaturation controller. Figure 4 shows the time-domain representation of the operating curves corresponding to Figure 3. Since the robust profile operates at lower supersaturation the cooling is slower than in the nominal case resulting in longer batch time for similar yield. The 
robust operating policy also indicates slower cooling and even a slight increase in the temperature during the initial part of the batch when the nuclei are generated. This is in correlation with the often used industrial practice, according to which slow cooling and moderate increase in temperature after the onset of nucleation can result in reduced variability in the final CSD. Monte Carlo simulations were performed by randomly sampling (100 samples) the uncertain parameter space $\theta_{b}$ and applying the nominal and robust temperature profiles. Figure 5 demonstrates that the robust operating curve leads to significantly reduced variability in the product quality compared to the nominal operating policy. Note that in practice often seeding and operating far from the uncertain nucleation zone is used to avoid the variability in the product CSD due to uncertainties in the primary nucleation kinetics. This generally leads to conservative operating curves with increased batch times due to the operation at low supersaturation. Additionally often the generation of high quality seed with consistent CSD is very difficult and variations in the seed CSD may result in larger variability of the product CSD. Conceptually un-seeded operation (i.e. in situ seed generation) would be the preferred operation for crystallisation systems. However in practice whether seeded or un-seeded operation would be applied depends on the particular system and whether the generation of consistent seed or controlled primary nucleation is the easier task in an industrial environment.

In the production of crystalline products often a desired dissolution profile (for example to achieve constant concentration level of pharmaceutical ingredients for a longer period) can be achieved by obtaining a desired multimodal CSD. To test the performance of the approach for crystalline product design with tailored dissolution profile the optimization was repeated for the case of a bimodal target distribution given as the weighted sum of two lognormal distributions,

$$
f_{n}^{\text {desired }}(L)=\frac{1}{\sqrt{2 \pi} L}\left(w \frac{1}{\sigma_{1}} \exp \left(-\frac{1}{2} \frac{\left(\ln (L)-\bar{L}_{1}\right)^{2}}{\sigma_{1}^{2}}\right)+(1-w) \frac{1}{\sigma_{2}} \exp \left(-\frac{1}{2} \frac{\left(\ln (L)-\bar{L}_{2}\right)^{2}}{\sigma_{2}^{2}}\right)\right),
$$

with $\bar{L}_{1}=-6, \sigma_{1}=0.22, \bar{L}_{2}=-7, \sigma_{2}=0.1$, and $w=0.15$ with the distribution in $\mathrm{m}^{-1}$.

Figure 6 shows the desired distribution at the end of the batch and the results of the Monte Carlo simulations corresponding to the nominal and robust operating profiles shown in Figure 7 in the phase diagram and in Figure 8 in time domain, respectively. The same initial distribution was used as in the 
previous case. The trajectories during the first part of the batch are very similar to those obtained for the monomodal CSD target. To obtain the bimodal distribution the controller drives the process to cross the nucleation curve for the second time. In both cases similar crystallization yield is obtained. The robust operating profile results in longer batch time. The consistency of the product CSD is significantly better for the robust temperature trajectory than for the nominal operating curve.

The performance of the batch NMPC approach is tested in the case of sudden change in the nucleation curve, which was simulated experimentally by introducing a certain amount of fine particles in the system. Similar scenarios may happen in practice due to accidental seeding, resulting from crusting or imperfectly cleaned crystallization vessel. The hierarchical implementation of the approach, which consists of the batch NMPC at the higher level and the supersaturation controller (SSC) at the lower level, is shown in Figure 9. For the control the batch time was divided in 20 discrete intervals and the temperature profile was approximated using a piecewise linear function. The NMPC uses concentration, temperature and CSD measurements to repeatedly calculate the operating profile $C_{s e t}=f(T)$ in the phase diagram, which is sent as a setpoint to the SSC. An estimator can be used when full CSD measurement data is not available. The focused beam reflectance measurement (Lasentec FBRM) from Mettler-Toledo with a conversion algorithm to transform the chord length distribution into CSD is an ideal PAT tool which can provide in situ full CSD information in real-time. However a dynamic estimation of the conversion shape parameter has been implemented using a moving horizon estimation type approach, which adapted the conversion shape factor to minimize the error between the predicted CSD and the CSD obtained from the chord length distribution conversion. The concentration measurements were performed using ATR-UV/Vis spectroscopy coupled with robust chemometrics. In the implemented configuration a full state feedback was possible and the estimator was used to enhance the convergence of the chord length distribution (CLD) to CSD conversion and decrease the effect of model plant mismatch. In the ideal case the operating profile resulted from the batch NMPC is equivalent to the one obtained from the nominal or robust open-loop optimization, and the NMPC algorithm provides very similar trajectory from one step to the other according to a shrinking horizon approach. When a disturbance is detected the NMPC altered the 
operating profile to correct the predicted effect of the disturbance on the final CSD. Figure 10 shows the operating profiles obtained from the NMPC.

When the shift in the nucleation curve occurs continuing the original operating curve results in excessive undesired nucleation. The NMPC drives the process in the undersaturated region by increasing the temperature. This dissolves the particles resulted from the unwanted second nucleation event. Figure 11 shows that the batch NMPC is able to achieve the desired target CSD despite the second nucleation event. When the original nominal profile is implemented it leads to significant secondary nucleation, which results in smaller average size and bimodal CSD. The resulted temperature profile is significantly faster (Figure 10B) in the nominal case since the excessive nucleation consumes the solute from the solution faster.

\section{Conclusions}

The paper presents a novel hierarchical robust control approach for the design of crystalline products by shaping the crystal size distribution. A distributional optimization approach is used to design a robust concentration versus temperate profile, which is used as a setpoint for a lower level supersaturation controller. The population balance model is solved using a novel combined quadrature method of moment and method of characteristics approach.

\section{Acknowledgements}

Financial support by EPSRC (EP/E022294/1) and the Royal Society of Engineering, U.K. is gratefully acknowledged.

\section{References}

Aamir, E., Nagy, Z. K., Rielly, C. D., Kleiner, T., Judat, B. (2008). Efficient crystal size distribution estimation approach for growth dominated crystallization processes, in Proc. of the 17th International Symposium on Industrial Crystallization (ISIC 17), Maastricht, The Netherlands, Vol.3, 1733-1740. 
Barrett , P., Glennon, B. (2002), Characterizing the Metastable Zone width and solubility curve using Lasentec FBRM and PVM, Chemical Engineering Research and Design, 80, 799-805.

Barrett, P., Smith, B., Worlitschek, J., Bracken, V., O'Sullivan, B., O'Grady, D. (2005), A review of the use of process analytical technology for the understanding and optimization of production batch crystallization processes, Organic Process Research \& Development, 9, 348-355.

Biegler, L., J. Rawlings (1991). Optimization Approaches to Nonlinear Model Predictive Control, Chemical Process Control, South Padre Island, TX, 1991.

Braatz, R.D. (2002). Advanced control of crystallization processes, Annual Reviews in Control, 26, $87-99$

Braatz, R. D., Hasebe, S. (2002), Particle size and shape control in crystallization processes, AIChE symp Series, 98, 307-327.

Diehl, M., H.G. Bock, J.P. Schlöder, R. Findeisen, Z.K. Nagy, F. Allgöwer (2002). Real-time optimization and nonlinear model predictive control of processes governed by differential-algebraic equations, J. of Process Control 12, 577-585.

Franke, R., E. Arnold, H. Linke. HQP: A solver for nonlinearly constrained optimization. http://hqp.sourceforge.net.

Fujiwara, M., Chow, P. S., Ma, D. L. and Braatz, R. D. (2002), Paracetamol crystallization using laser backscattering and ATR-FTIR spectroscopy: Metastability, agglomeration, and control, Crystal Growth \& Design 2 (5), 363-370.

Fujiwara, M., Z.K. Nagy, J.W. Chew, R.D. Braatz (2005). First-principles and direct design approaches for the control of pharmaceutical crystallization, J. of Process Control 15, 493-504.

Garside, J. (1984), Advances in characterization of crystal growth, in G. R. Youngquist (editor), Advances in Crystallization from Solutions, AIChE Symposium Series, Vol. 80, No. 240, AIChE Press, New York, 23-38

Hermanto, M. W., X. Y. Woo, R. D. Braatz, and M.-S. Chiu (2007), Robust optimal control of polymorphic transformation in batch crystallization, AIChE Journal, 53, 2643-2650.

Hounslow, M. J., G. K. Reynolds (2006), Product engineering for crystal size distribution, AIChE Journal, 52, 2507-2517. 
Jones, A.G. (2002), Crystallization Process Systems, Oxford: Butterworth-Heinemann.

Larsen, P. A., Patience, D. B. and Rawlings, J. B. (2006), Industrial crystallization process control, IEEE Control Systems Magazine, 26 (4), 70-80.

LeVeque, R. (1992). Numerical Methods for Conservation Laws, Birkhauser.

McGraw, R. (1997). Description of atmospheric aerosol dynamics by the quadrature method of moments, Aerosol Science and Technology 27, 255-265.

Nagy, Z.K., F. Allgower, R. Franke, A. Frick, B. Mahn (2004). Efficient tool for nonlinear model predictive control of batch processes, in Proc. of the 12th Mediter. Conf. on Cont. and Autom., Kusadasi, Turkey.

Nagy, Z. K., R. D. Braatz (2004), Open-loop and closed-loop robust optimal control of batch processes using distributional and worst-case analysis, Journal of Process Control, 14, 411-422.

Nagy, Z.K., R.D. Braatz (2003). Robust nonlinear model predictive control of batch processes, AIChE J. 49, 1776-1786.

Nagy, Z.K., J.W. Chew, M. Fujiwara, and R. D. Braatz (2008), Comparative performance of concentration and temperature controlled crystallizations, Journal of Process Control, 18 (3-4), $399-407$.

Nagy, Z. K., B. Mahn, R. Franke, F. Allgower (2007), Efficient output feedback nonlinear model predictive control for temperature control of industrial batch reactors, Control Engineering Practice, 15, 839-859.

Nyvlt, J., O. Sohnel, M. Matuchova, and M. Broul (1985), The kinetics of industrial crystallization, Elsevier, Amsterdam.

Rawlings, J., S. Miller, W. Witkowski (1993). Model identification and control of solution crystallization processes A review, Ind. Eng. Chem. Res. 32, 1275-1296.

Sarkar, D., Rohani, S. and Jutan, A. (2006), Multi-objective optimization of seeded batch crystallization processes, Chemical Engineering Science, 61 (16): 5282-5295

Sheikhzadeh, M., Trifkovic M. and Rohani, R. (2008a), Fuzzy logic and rigid control of a seeded semi-batch, anti solvent, isothermal crystallizer, Chemical Engineering Science, 63 (4), 991-1002 
Sheikhzadeh, M., Trifkovic, M. and Rohani, R. (2008b), Adaptive MIMO neuro-fuzzy logic control of a seeded and an unseeded anti-solvent semi-batch crystallizer, Chemical Engineering Science, 63(5), 1261-1272.

Ward, J. D., Mellichamp, D. A. and Doherty, M. F. (2006)1, Choosing an operating policy for seeded batch crystallization, AIChE Journal 52 (6), 2046-2054.

Wibowo, C., W.-C. Chang, et al. (2001). Design of integrated crystallization systems, AlChE Journal 47 (11), 2474-2492.

Worlitschek, J., Mazzotti, M. (2004), Model-based optimization of particle size distribution in batchcooling crystallization of paracetamol, Crystal Growth \& Design, 4 (5), 891-903.

Yu, L. X., Lionberger, R. A., Raw, A. S., D'Costa, R., Wu, H. Q., Hussain, A. S. (2004), Applications of process analytical technology to crystallization processes, Advanced Drug Delivery Reviews, 56 $349-369$

Zhou, G.X., Fujiwara, M., Woo, X.Y., Rusli, E., Tung, H.H., Starbuck, C., Davidson, O., Ge, Z.H., Braatz, R.D. (2006), Direct design of pharmaceutical antisolvent crystallization through concentration control. Crystal Growth \& Design 6 (2006) 892-898. 


\section{Figure Caption}

Fig. 1. Operations of seeded and unseeded batch cooling crystallizers.

Fig. 2. Main idea of the shrinking horizon batch NMPC.

Fig. 3. Phase diagram with nominal $(w=0)$ and robust ( $w=0.3)$ operating curves for monomodal target CSD.

Fig. 4. Time-domain representation of the nominal and robust operating profiles corresponding to Figure 3.

Fig. 5. Monomodal target and product CSDs resulting from Monte Carlo simulations with the uncertain nucleation parameters using the nominal and robust operating profiles.

Fig. 6. Bimodal target and product CSDs resulting from Monte Carlo simulations with the uncertain nucleation parameters using the nominal and robust operating profiles.

Fig. 7. Phase diagram with nominal $(w=0)$ and robust $(w=0.3)$ operating curves for the bimodal target CSD.

Fig. 8. Time-domain representation of the nominal and robust operating profiles for the bimodal target CSD.

Fig. 9. Architecture for robust control of the shape of CSD for batch cooling crystallization.

Fig. 10. Phase-diagram (A) and time-domain (B) representation of the nominal and batch NMPC profiles in the case of shift in the nucleation curve.

Fig. 11. Target and product CSD at the end of the batch for nominal operating curve and batch NMPC. 


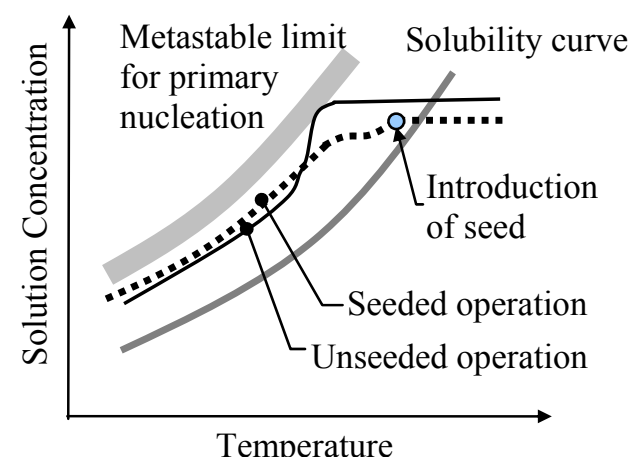

Fig. 1. Operations of seeded and unseeded batch cooling crystallizers. 


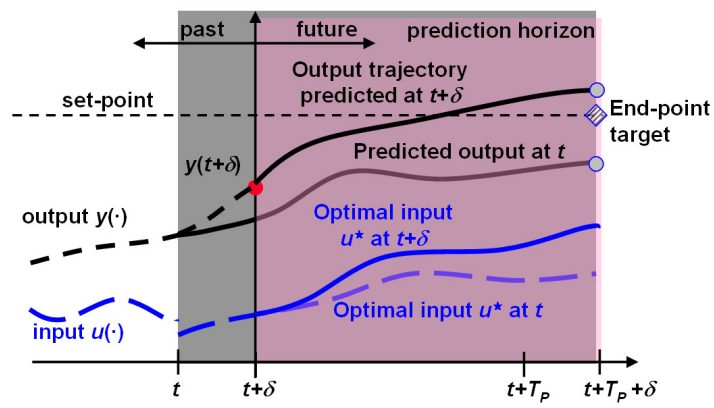

Fig. 2. Main idea of the shrinking horizon batch NMPC. 


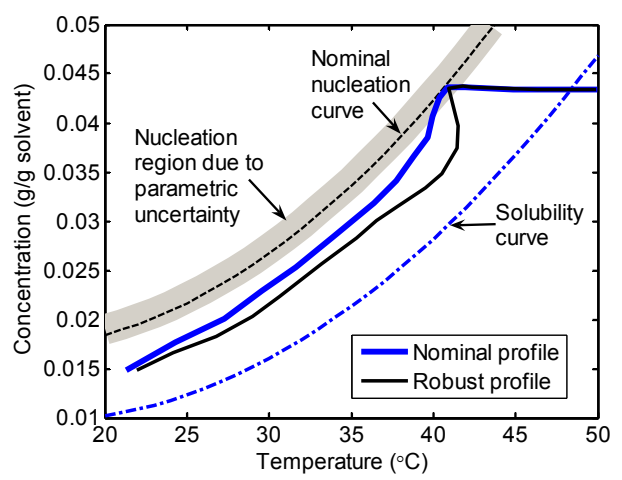

Fig. 3. Phase diagram with nominal $(w=0)$ and robust $(w=0.3)$ operating curves for monomodal target CSD. 


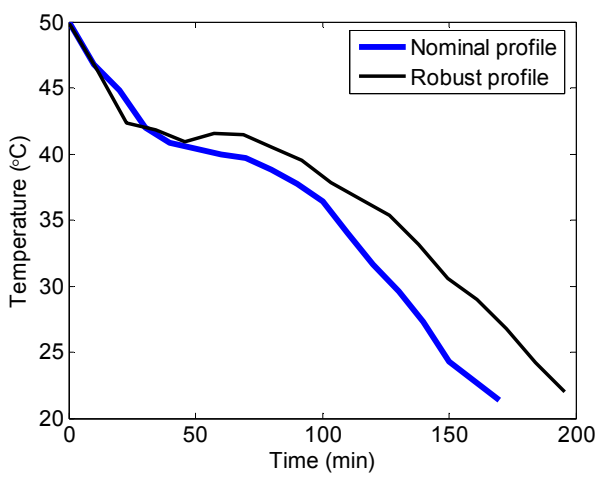

Fig. 4. Time-domain representation of the nominal and robust operating profiles corresponding to Figure 3. 




Fig. 5. Monomodal target and product CSDs resulting from Monte Carlo simulations with the uncertain nucleation parameters using the nominal and robust operating profiles. 


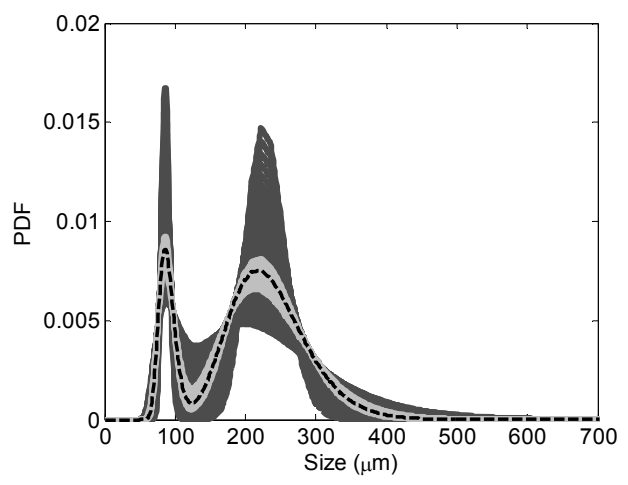

Fig. 6. Bimodal target and product CSDs resulting from Monte Carlo simulations with the uncertain nucleation parameters using the nominal and robust operating profiles. 


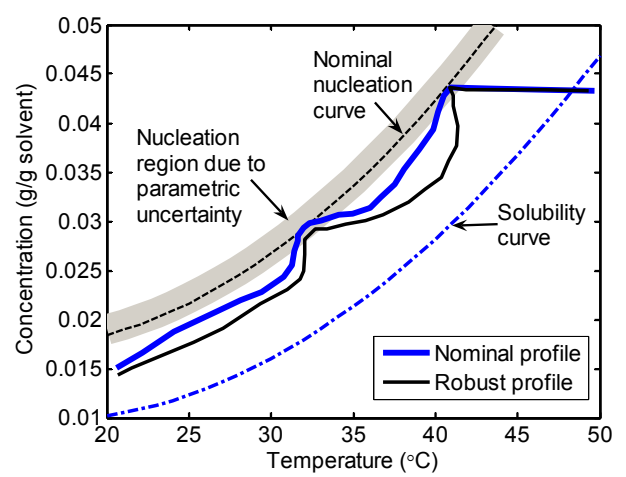

Fig. 7. Phase diagram with nominal $(w=0)$ and robust $(w=0.3)$ operating curves for the bimodal target CSD. 


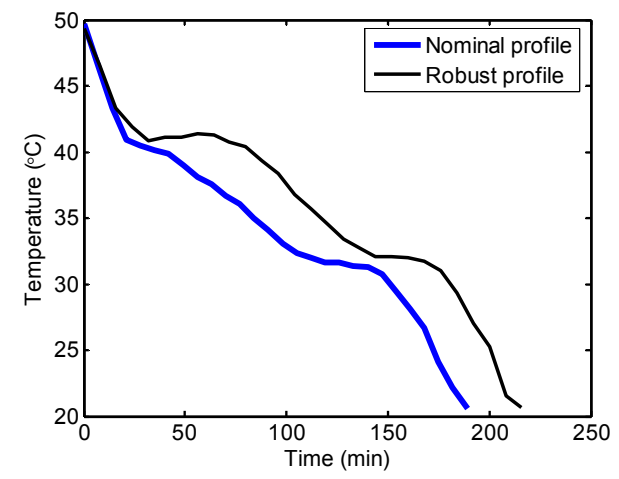

Fig. 8. Time-domain representation of the nominal and robust operating profiles for the bimodal target CSD. 


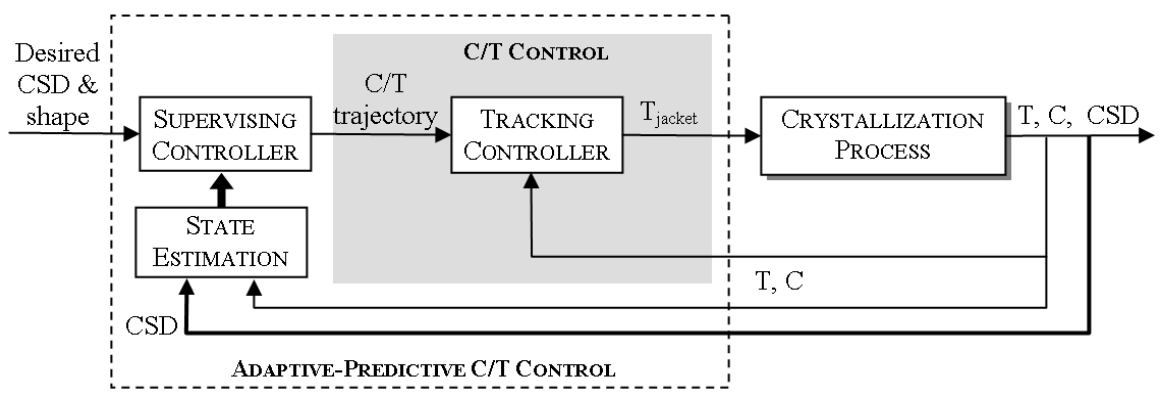

Fig. 9. Architecture for robust control of the shape of CSD for batch cooling crystallization. 

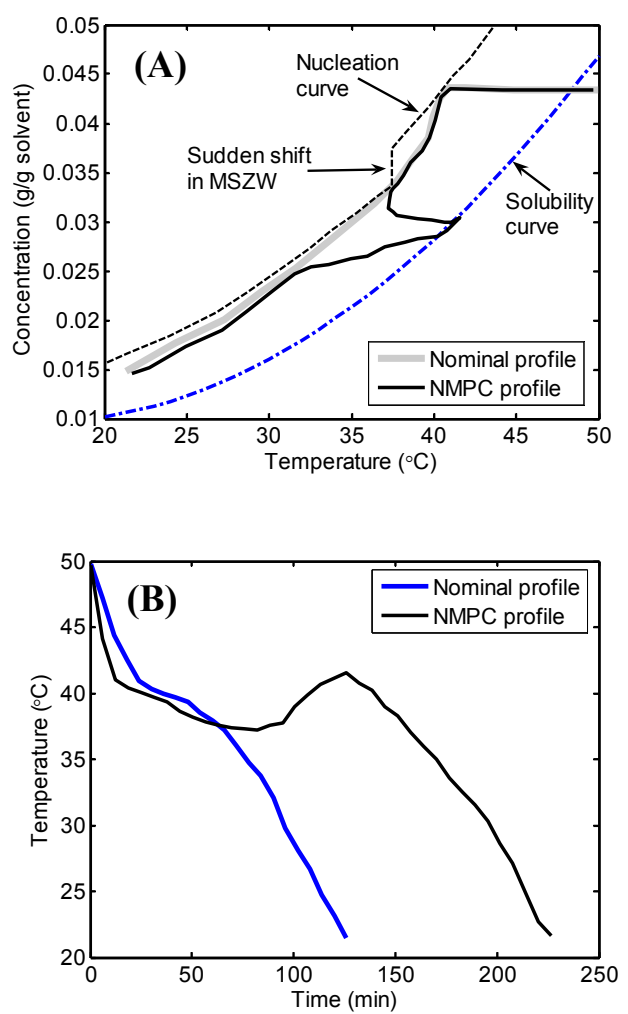

Fig. 10. Phase-diagram (A) and time-domain (B) representation of the nominal and batch NMPC profiles in the case of shift in the nucleation curve. 


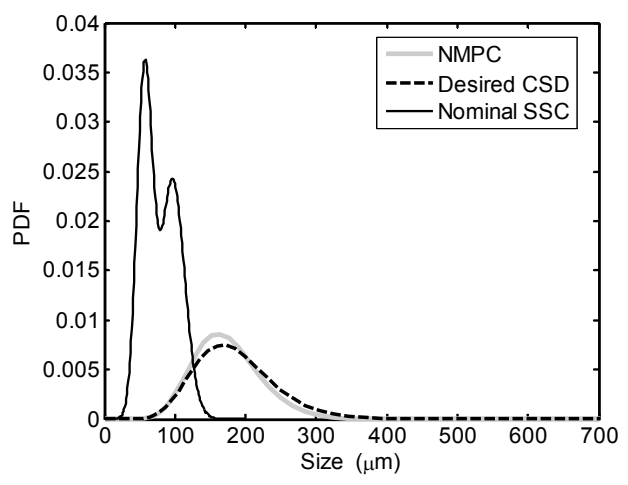

Fig. 11. Target and product CSD at the end of the batch for nominal operating curve and batch NMPC. 